\title{
Evaluation of an inflammation-based prognostic score for the identification of patients requiring postoperative adjuvant chemotherapy for stage II colorectal cancer
}

\author{
YUJI TOIYAMA, CHIKAO MIKI, YASUHIRO INOUE, KOJI TANAKA, \\ YASUHIKO MOHRI and MASATO KUSUNOKI
}

Department of Gastrointestinal and Pediatric Surgery, Mie University Graduate School of Medicine, Mie 514-8507, Japan

Received September 28, 2010; Accepted November 30, 2010

DOI: $10.3892 / \mathrm{etm} .2010 .175$

\begin{abstract}
Recent studies have revealed the Glasgow prognostic score (GPS) to aid in the prediction of postoperative outcome in colorectal cancer patients. However, whether GPS predicts poor prognosis in curative colorectal cancer patients has yet to be ascertained. Furthermore, there is no information on the association between GPS and adjuvant chemotherapy in stage II or III colorectal cancer patients. A total of 219 patients with stage II and III colorectal cancer were included in this trial. The modified GPS (mGPS) defined in this study was calculated on the basis of admission data as follows: patients with an elevated level of both C-reactive protein $(0.5 \mathrm{mg} / \mathrm{dl})$ and hypoalbuminemia (Alb $<3.5 \mathrm{mg} / \mathrm{dl}$ ) were allocated a score of 2 , and patients showing 1 or none of these blood chemistry abnormalities were allocated a score of 1 or 0 , respectively. The association between the mGPS and clinicopathological findings and survival was retrospectively assessed. The mGPS was significantly higher in patients with an advanced age, serosal invasion, advanced stage cancer and pre-operative high CEA levels. Kaplan-Meier analysis revealed that a higher GPS predicted a higher risk of postoperative mortality in stage II and/or III colorectal cancer patients. Multivariate analyses revealed that the mGPS was the most sensitive predictor of postoperative mortality in stage II/III or stage II, respectively. The prognosis of stage II patients with a higher mGPS was as favorable as that of patients with a lower mGPS when adjuvant chemotherapy was undertaken. Pre-operative mGPS is considered to be a useful predictor of postoperative mortality in patients with stage II and/or III colorectal cancer, independently of the CEA test or TNM system. Postoperative adjuvant chemotherapy may be recommended for stage II colorectal cancer patients with a high mGPS.
\end{abstract}

Correspondence to: Dr Yuji Toiyama, Department of Gastrointestinal and Pediatric Surgery, Mie University Graduate School of Medicine, 2-174 Edobashi, Tsu, Mie 514-8507, Japan

E-mail: ytoi0725@clin.medic.mie-u.ac.jp

Key words: colorectal cancer, Glasgow prognostic score, stage II, prognosis, chemotherapy

\section{Introduction}

Colorectal cancer is the most common malignant disease worldwide. Despite potentially curative surgery, approximately $30 \%$ of patients develop metastases, even when adjuvant therapies, inclusing chemotherapy and radiochemotherapy, are administered (1). Although adjuvant chemotherapy provides a significant survival benefit in stage III patients, whether this treatment has any effect on patients with stage II colon cancer remains controversial, since $20-30 \%$ of these patients eventually experience tumor relapses (2). Adjuvant chemotherapy was shown to increase the survival of certain populations of stage II patients (3). Furthermore, it was found that $60 \%$ of stage III patients did not relapse, even when adjuvant chemotherapy was not used (4). Therefore, identifying high-risk patients among those with stage II or III colorectal cancer will aid in the selection of candidates for standard or intensive adjuvant therapy.

C-reactive protein (CRP) is an acute phase reactant that acts as a surveillance molecule for the activation of the adaptive immune system. It is synthesized in hepatocytes and is up-regulated by cytokines such as interleukin (IL)-6 and tumor necrosis factor- $\alpha$ (33). McMillan et al, among others, showed that elevated CRP levels are associated with an increased risk for early recurrence and a poor outcome following colorectal cancer surgery (5-11).

We previously reported that CRP levels reflect IL-6 production in colorectal cancer tissues and predict poor prognosis in colorectal cancer patients, particularly in stage I or II patients who are not usually candidates for postoperative adjuvant chemotherapy $(12,13)$. It is conceivable that the pre-operative presence of an acute phase reactant adversely affects prognosis, since IL-6, for example, acts as a potent tumor growth factor (14).

By contrast, it has long been recognized that low circulating albumin concentrations prior to surgery are associated with a poor outcome in patients with colorectal cancer (15-17). For example, Heys et al (16) showed that low concentrations of circulating albumin before surgery and the magnitude of the decrease were associated with poor overall survival.

Therefore, it is of interest that the combination of hypoalbuminemia and elevated CRP levels, as in the Glasgow prognostic score (GPS), have been shown to provide addi- 

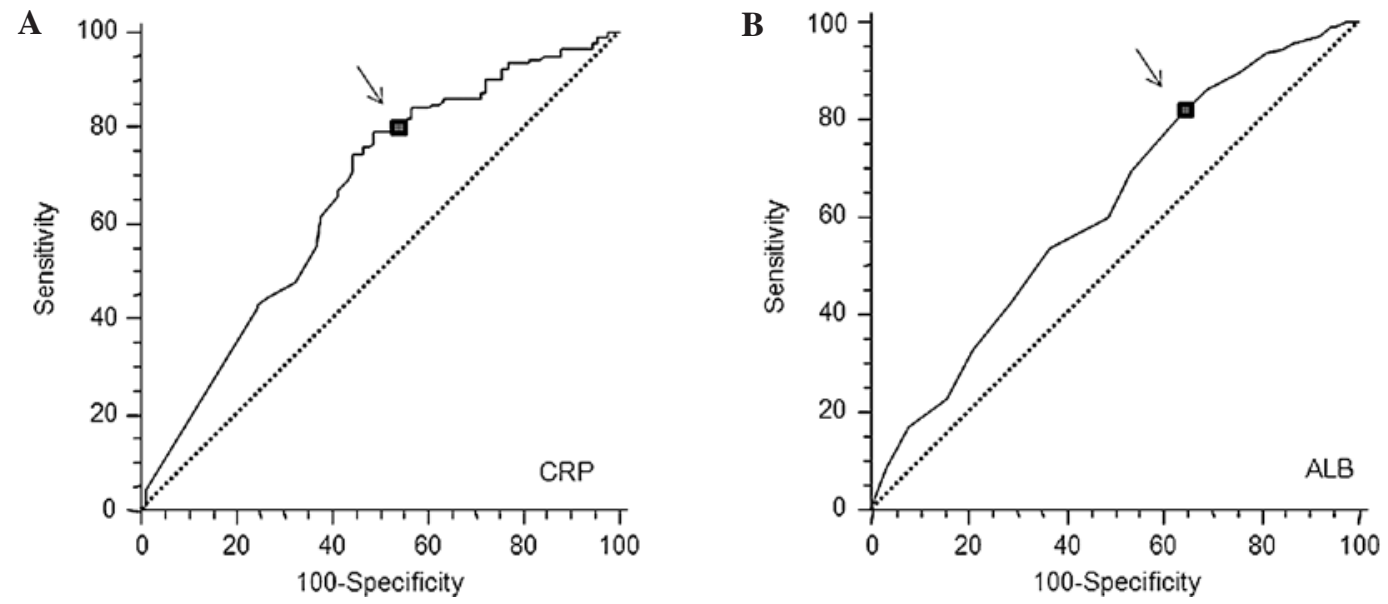

Figure 1. Receiver operating characteristic (ROC) curves for (A) peak serum CRP and (B) albumin (ALB) in patients with stage II and III colorectal cancer $(\mathrm{n}=219)$. The arrows indicate the location on the ROC curves for the diagnostic cut-off point that minimizes the misclassification of surviving and deceased patients. CRP: sensitivity $79.5 \%$, specificity $51.1 \%$, cut-off $0.5 \mathrm{mg} / \mathrm{dl}$. ALB: sensitivity $80.7 \%$, specificity $36.7 \%$, cut-off $3.5 \mathrm{mg} / \mathrm{dl}$.

tional prognostic information for patients with advanced cancer in various organs, including colorectal cancer (18-23). Furthermore, the GPS was recently validated as a prognostic score for patients undergoing potentially curative resection for stage II or stage III colon cancer (24). However, associations between the GPS and adjuvant chemotherapy in stage II or III colorectal cancer patients have yet to be investigated.

Therefore, in this study we first examined, using the GPS, whether the combination of an elevated CRP and hypoalbuminemia could identify a subset of stage II or III colorectal cancer patients with a poor prognosis who require postoperative adjuvant therapy. Second, we evaluated whether the use of adjuvant chemotherapy improves the survival of patients with a poor prognosis as predicted by the GPS.

\section{Materials and methods}

Overall, 219 patients with stage II or III colorectal cancer who received potentially curative surgery at our institution between January 1995 and January 2005 were enrolled in this retrospective study. Curative resection was defined as the absence of any gross residual tumor from the surgical bed and a surgical resection margin that was pathologically negative for tumor invasion. Data were retrieved from operative and pathological reports. Follow-up data were obtained from the outpatient clinical database.

The study group comprised 136 men and 83 women aged 29-91 years (median 66; interquartile range 58-73). Staging was principally based on the UICC/TNM classification of colorectal cancer. Overall, 125 patients had stage II and 94 had stage III disease. Experienced pathologists from our institution participated in this study and verified the accuracy of the original diagnosis. Of the 219 registered patients, $110 \mathrm{had}$ tumors located in the colon and 109 had tumors located in the rectum. The pathologic tumor diameter indicated the maximum microscopic length of the tumor, irrespective of the depth. Differentiated tumors were histologically observed in 200 patients and undifferentiated tumors in 19 patients. Lymphatic invasion was observed in 191 patients and vascular invasion in 96 patients. After 1997, 96 patients with a favorable performance status who gave informed consent received adjuvant chemotherapies. Starting 4 weeks after curative surgery, pyrimidine-fluoride-based regimens were administered for 0.5-1 years to patients classified as mainly stage III [stage II, 45/125 (36\%); stage III, 51/94 (54.3\%)].

The patients were followed up every 12-16 weeks for at least 5 years according to our standard protocol, which included tumor-marker studies, computed tomography, colorectal fiber examinations, ultrasonography and chest radiography. Bone scans were performed when bone metastasis was indicated. The median follow-up time was 52.7 months (mean 56.9 \pm 63.8 ). The clinicopathologic parameters studied for prognostic value were tumor size, $\mathrm{T}$ classification, vessel involvement, lymphatic invasion, lymph node metastasis and serum carcinoembryonic antigen (CEA) concentration.

Blood samples were taken for routine laboratory measurements of albumin and CRP before surgery. This is the standard practice for all patients with cancer in our institution. The coefficient of variation for these methods, over the range of measurement, was $<5 \%$, as established by routine quality control procedures. Patients who underwent non-elective surgery or pre-operative radiotherapy, succumbed within 30 days of surgery or showed clinical evidence of infection or other inflammatory conditions, were excluded from the study.

The GPS was determined as previously described (18). Briefly, patients with elevated CRP levels ( $>1 \mathrm{mg} / \mathrm{dl})$ plus hypoalbuminemia $(<3.5 \mathrm{~g} / \mathrm{dl})$ were allocated a score of 2 (positive). Patients with only one of these factors were allocated a score of 1 (positive). Patients with neither of these factors were allocated a score of 0 (negative). In the present study, elevated serum CRP and lower albumin levels were defined according to the best predictive values calculated by receiver operating characteristic (ROC) analyses, which found the best pair of values for highest sensitivity and highest specificity based on the peak and cut-off points (Fig. 1A and B). Based on this analysis, the cut-off for CRP was calculated to be $0.5 \mathrm{mg} / \mathrm{dl}$ and that for hypoalbuminemia was unchanged $(3.5 \mathrm{mg} / \mathrm{dl})$. This modified GPS (mGPS) was also used, and patients with elevated CRP levels $(>0.5 \mathrm{mg} / \mathrm{dl})$ and hypoalbuminemia $(<3.5 \mathrm{~g} / \mathrm{dl})$ were allocated a score of 2 (mGPS 2). Patients with only one factor 
Table I. Association between GPS and clinicopathological characteristics of stage II and III patients undergoing potentially curative resection for colon cancer.

\begin{tabular}{|c|c|c|c|c|c|}
\hline & Patients & GPS 0 & GPS 1 & GPS 2 & p-value \\
\hline Age (years) & & & & & 0.0080 \\
\hline$\leq 64$ & 95 & 72 & 17 & 6 & \\
\hline$>64$ & 124 & 70 & 34 & 20 & \\
\hline Gender & & & & & 0.9700 \\
\hline Female & 83 & 53 & 20 & 10 & \\
\hline Male & 136 & 89 & 31 & 16 & \\
\hline $\begin{array}{l}\text { Vascular } \\
\text { invasion }\end{array}$ & & & & & 0.6320 \\
\hline No & 123 & 83 & 27 & 13 & \\
\hline Yes & 96 & 59 & 24 & 13 & \\
\hline $\begin{array}{l}\text { Lymphatic } \\
\text { invasion }\end{array}$ & & & & & 0.4670 \\
\hline No & 28 & 21 & 5 & 2 & \\
\hline Yes & 191 & 121 & 46 & 24 & \\
\hline $\begin{array}{l}\text { Serosal } \\
\text { invasion }\end{array}$ & & & & & 0.0040 \\
\hline No & 139 & 100 & 29 & 10 & \\
\hline Yes & 80 & 42 & 22 & 16 & \\
\hline Pathology & & & & & 0.9430 \\
\hline Diff. & 200 & 129 & 47 & 24 & \\
\hline Non diff. & 19 & 13 & 4 & 2 & \\
\hline TNM stage & & & & & 0.0130 \\
\hline II & 125 & 89 & 20 & 16 & \\
\hline III & 94 & 53 & 31 & 10 & \\
\hline Chemotherapy & & & & & 0.5700 \\
\hline No & 123 & 77 & 29 & 17 & \\
\hline Yes & 96 & 65 & 22 & 9 & \\
\hline CEA & & & & & 0.0002 \\
\hline$\leq 6$ & 134 & 100 & 26 & 8 & \\
\hline$>6$ & 85 & 42 & 25 & 18 & \\
\hline
\end{tabular}

GPS, Glasgow prognostic score; CEA, carcinoembryonic antigen.

were allocated a score of 1 (mGPS 1 ) and patients with neither factor were allocated a score of 0 (mGPS 0 ).

Statistical methods. Data are presented as the means \pm standard deviation (SD). Comparisons were made using the non-parametric Mann-Whitney $U$ test for continuous variables and the Chi-square test for categorical data. The correlations were analyzed by Spearman's coefficient analysis. ROC analyses were performed to calculate the cut-off values according to the most accurate value obtained using Medcalc 7.2 for Windows (Mariakerke, Belgium). The survival probabilities were calculated using the product limit method of the Kaplan-Meier methods, considering treatment- and colorectal cancer-related mortality. The differences between two groups were determined using the log-rank test. The influence of each significant predictor identified by log-rank tests was assessed by multivariate analysis using Cox's proportional
Table II. Clinicopathological characteristics of stage II and III patients undergoing potentially curative resection for colorectal cancer: univariate survival analysis.

\begin{tabular}{|c|c|c|c|}
\hline & HR & $95 \% \mathrm{CI}$ & p-value \\
\hline Age $(\leq 64$ vs. $>64)$ & 1.02 & $0.530-1.95$ & 0.9500 \\
\hline Gender (male vs. female) & 1.21 & $0.610-2.44$ & 0.5600 \\
\hline Chemotherapy (yes vs. no) & 1.04 & $0.540-2.01$ & 0.9000 \\
\hline Venous invasion (yes vs. no) & 1.17 & $0.600-2.32$ & 0.6300 \\
\hline Lymphatic invasion (yes vs. no) & 1.15 & $0.430-3.05$ & 0.8000 \\
\hline $\begin{array}{l}\text { Lymph-node metastasis } \\
\text { (yes vs. no) }\end{array}$ & 1.45 & $0.760-2.86$ & 0.2500 \\
\hline Serosal invasion (yes vs. no) & 1.99 & $1.060-4.12$ & 0.0300 \\
\hline Pathology (well vs. poor diff.) & 0.33 & $0.046-0.58$ & 0.0050 \\
\hline $\mathrm{CEA}(\leq 6 \mathrm{vs} .>6)$ & 2.66 & $1.460-5.63$ & 0.0020 \\
\hline mGPS (0 vs. 1.2) & 0.33 & $0.140-0.58$ & 0.0005 \\
\hline
\end{tabular}

mGPS, modified Glasgow prognostic score; CEA, carcinoembryonic antigen.

hazards model. The statistical analyses were carried out using StatView 5.0 (SAS Institute Inc., Cary, NC, USA) for Windows. Two-sided p-values of $<0.5$ were considered statistically significant.

\section{Results}

Association between the mGPS and the clinicopathological characteristics of patients undergoing potentially curative resection for colorectal cancer. During the observation period, 37 patients succumbed to colorectal cancer. Overall, 57 patients had elevated CRP levels $(>0.5 \mathrm{mg} / \mathrm{dl})$ and $46 \mathrm{had}$ hypoalbuminemia $(<3.5 \mathrm{mg} / \mathrm{dl})$. Of the 57 patients with elevated CRP levels, 26 (45.6\%) also had hypoalbuminemia.

Table I shows the relationship between clinicopathological characteristics and mGPS in patients with stage II and III colorectal cancer. Gender, vascular or lymphatic invasion, lymph node metastasis, pathological differentiation and postoperative adjuvant chemotherapy were not significantly associated with the mGPS classification. However, age, serosal invasion, CEA level and TNM classification were significantly associated with the mGPS classification.

Univariate and multivariate analyses in relation to mortality in patients with stage II or III colorectal cancer. The results of the univariate analysis of postoperative mortality, using the same factors as those in Table I, are presented in Table II. In our patients with stage II and III colorectal cancer, we defined patients with elevated mGPS as those with mGPS 1 and mGPS 2, according to the best predictive values calculated by ROC analyses, which found the best pair of values for highest sensitivity (69.23\%) and highest specificity $(56.76 \%)$ using a peak cut-off point (Fig. 3A). By contrast, the ROC curve in Fig. 3B for CEA in stage II and III colorectal cancer, showed that the best cut-off value to predict prognosis and area under curve (AUC) of CEA was inferior to that of mGPS (CEA 0.610; mGPS 0.635). 
A

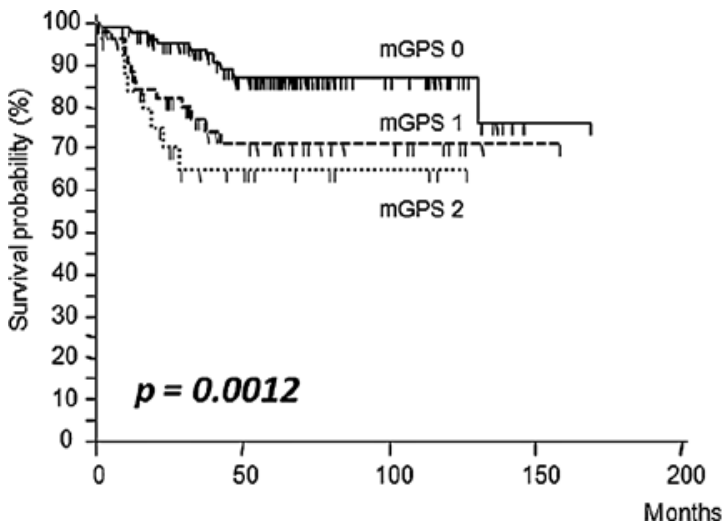

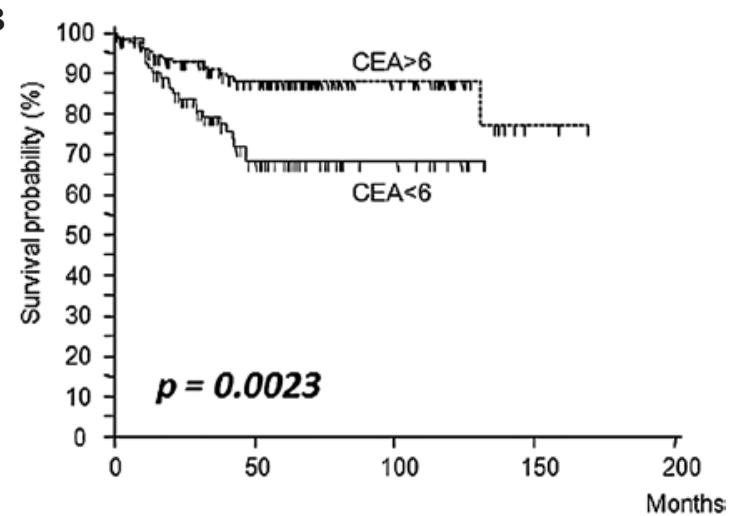

Figure 2. Kaplan-Meier analysis of the 5-year survival rates of stage II and III patients according to (A) inflammatory status-based prognostic score (mGPS 0 , 1 and 2), (B) with or without elevated serum CEA levels. mGPS, modified Glasgow prognostic score; CEA, carcinoembryonic antigen.

A

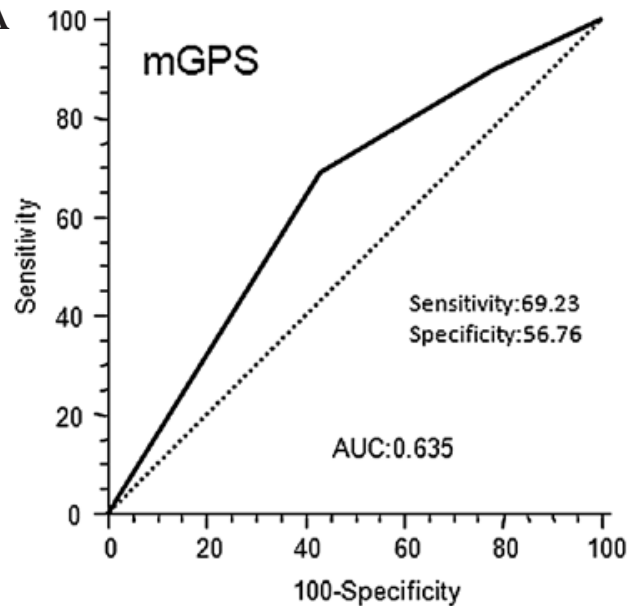

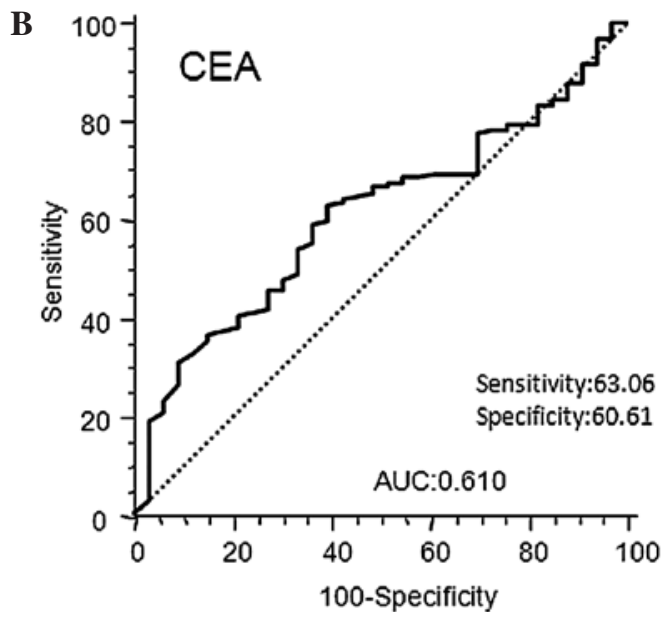

Figure 3. Receiver operating characteristic curves of (A) inflammatory status-based prognostic score (mGPS 0, 1 and 2) and (B) serum CEA levels in stage II and III colorectal cancer patients showing the best cut-off value to predict prognosis and AUC (CEA, 0.610; mGPS, 0.635). mGPS, modified Glasgow prognostic score; CEA, carcinoembryonic antigen; AUC, area under curve.

Fig. 2A and B shows the survival curves of patients subdivided on the basis of their mGPS $(0,1$ and 2$)$ and CEA ( $\leq 6$ vs. $>6 \mathrm{ng} / \mathrm{ml}$ ) levels. Patients with elevated mGPS and CEA levels had a significantly worse prognosis than patients whose levels were below the cut-off value (log-rank test, mGPS: $p=0.0012$; CEA: $p=0.0023)$. Based on Cox's univariate proportional hazards analysis, serosal invasion $(p=0.03)$, undifferentiated tumors (poorly differentiated and mucinous adenocarcinoma) $(p=0.005)$, elevated serum CEA levels $(p=0.002)$ and elevated mGPS (mGPS1 and 2) $(\mathrm{p}=0.0005)$ were significant prognostic factors for poor overall survival in patients with stage II and III colorectal cancer (Table II). Multivariate analysis revealed that undifferentiated tumors $(\mathrm{p}=0.013)$ and elevated mGPS ( $\mathrm{p}=0.003$ ) were the only independent risk factors for predicting poor prognosis (Table III).

Furthermore, in stage II or III colorectal cancer, elevated mGPS was associated with poor survival (stage II, $\mathrm{p}=0.0068$; stage III, $\mathrm{p}=0.034$ ) (Fig. 4A and B) and was the only independent prognostic factor in stage II patients $(\mathrm{p}=0.005)$ (Table IV).

Evaluation of whether adjuvant chemotherapy improves the survival of patients with poor prognosis with stage II colorectal cancer as predicted by $m G P S$. Fig. 5 shows that in stage II colorectal cancer, mGPS-positive patients had a significantly worse prognosis compared to mGPS-negative patients when adjuvant chemotherapy was not used (Fig. 5A). By contrast, chemotherapy improved cancer-specific survival, even in the mGPS-positive patients (Fig. 5B).

\section{Discussion}

The TNM staging system provides the most reliable information on prognosis and aids in the discrimination of patients with early stage disease from those with advanced stage disease. However, it is less accurate for predicting the prognosis of patients with an intermediate extent of tumor invasion. CEA is a complex glycoprotein that is up-regulated in approximately $90 \%$ of advanced colorectal cancers and contributes to the malignant characteristics of tumors (25). However, it is not useful in detecting asymptomatic cancer, as the sensitivity of CEA determination for early colorectal cancer is as low as $30-40 \%$ (26). Moreover, CEA is not significantly associated with survival in patients with stage I and II lesions, and CEA testing is relatively insensitive to tumors with local or peritoneal involvement (27). Therefore, the identification of sensitive 

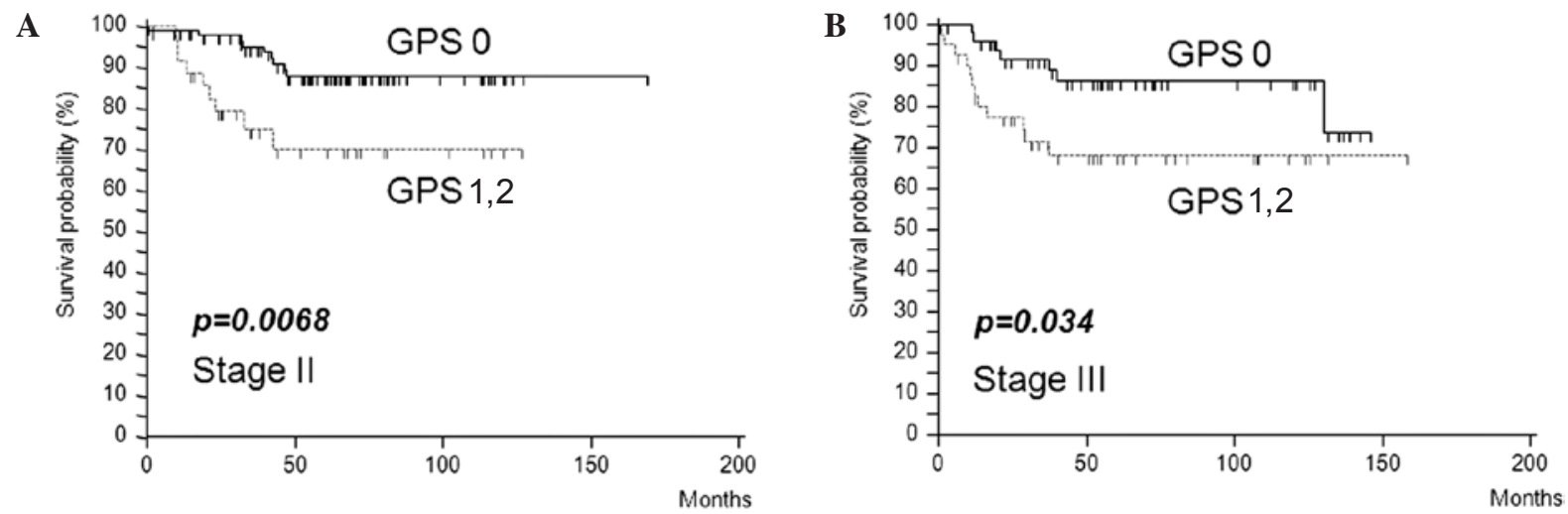

Figure 4. Kaplan-Meier analysis of the 5-year survival rates of patients with stage II (A) or stage III (B) colorectal cancer according to inflammatory statusbased prognostic score (mGPS 0 vs. mGPS 1/2).
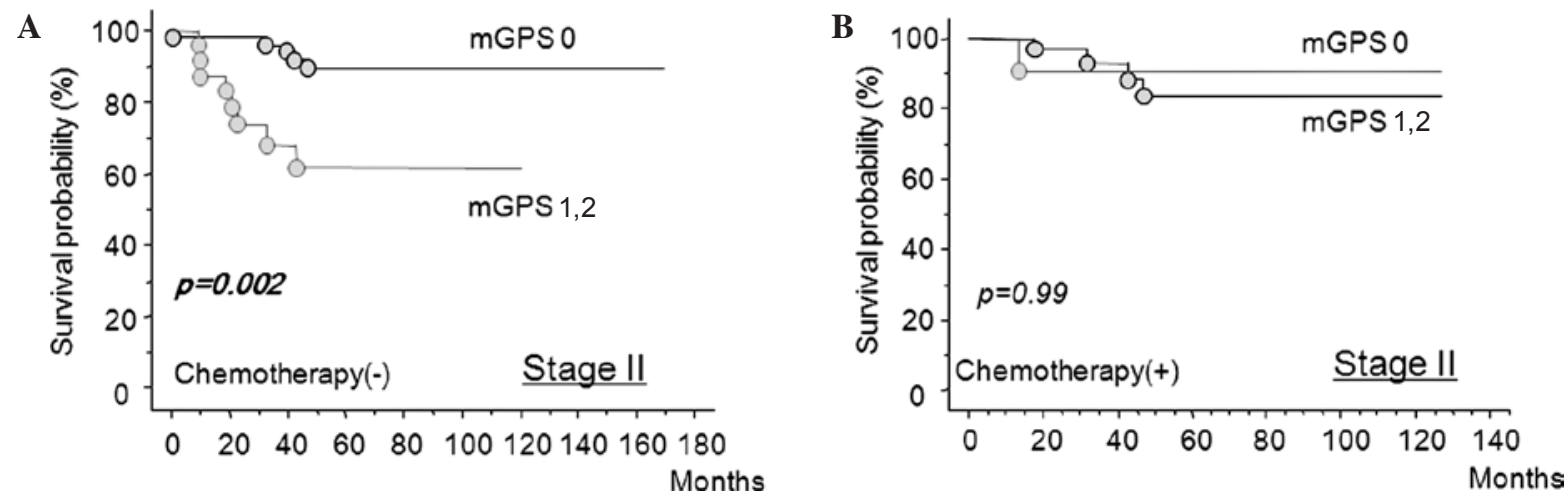

Figure 5. Evaluation of adjuvant chemotherapy in patients with stage II colorectal cancer who were predicted to have poor prognosis by mGPS. (A) Survival curves of stage II patients without adjuvant chemotherapy according to inflammatory status-based prognostic score (mGPS 0 vs. mGPS 1/2). (B) Survival curves of patients with stage II colorectal cancer treated with adjuvant chemotherapy according to inflammatory status-based prognostic score (mGPS 0 vs. mGPS $1 / 2$ ).

Table III. Clinicopathological characteristics of stage II and III patients undergoing potentially curative resection for colorectal cancer: multivariate survival analysis.

\begin{tabular}{lccc}
\hline & HR & $95 \%$ CI & p-value \\
\hline Serosal invasion (no vs. yes) & 0.75 & $0.38-1.47$ & 0.400 \\
Pathology (poor vs. well diff.) & 2.89 & $1.25-6.69$ & 0.013 \\
CEA ( $\leq 6$ vs. $>6)$ & 0.54 & $0.27-1.08$ & 0.080 \\
mGPS (1,2 vs. 0) & 2.80 & $1.43-5.49$ & 0.003 \\
\hline
\end{tabular}

prognostic markers in this subgroup will allow for the use of postoperative adjuvant therapy in a subset of patients with poor prognosis and improve survival.

Recently, the combination of hypoalbuminemia and elevated CRP levels (>1.0 mg/dl) (original GPS) has been shown to provide additional prognostic information for patients with curative or advanced colorectal cancer. In the present study, we defined that the cut-off value of CRP was $0.5 \mathrm{mg} / \mathrm{dl}$ according to the best predictive values calculated by ROC analyses (Fig. 1A). Furthermore, the classification of CRP using a cut-off value of $0.5 \mathrm{mg} / \mathrm{dl}$ discriminates highrisk patients more clearly than using $1.0 \mathrm{mg} / \mathrm{dl}$. CRP serum
Table IV.Clinicopathological characteristics and cancer-specific survival in patients undergoing potentially curative resection for stage II colorectal cancer: multivariate survival analysis.

\begin{tabular}{|c|c|c|c|}
\hline & HR & $95 \% \mathrm{CI}$ & p-value \\
\hline Age ( $\leq 64$ vs. $>64)$ & 0.56 & $0.19-1.70$ & 0.310 \\
\hline Gender (male vs. female) & 0.56 & $0.17-1.81$ & 0.330 \\
\hline Chemotherapy (yes vs. no) & 1.52 & $0.48-4.82$ & 0.480 \\
\hline Venous invasion (yes vs. no) & 0.64 & $0.19-2.11$ & 0.470 \\
\hline $\begin{array}{l}\text { Lymphatic invasion } \\
\text { (yes vs. no) }\end{array}$ & 2.02 & $0.45-8.18$ & 0.330 \\
\hline Serosal invasion (yes vs. no) & 0.93 & $0.33-2.63$ & 0.890 \\
\hline Pathology (well vs. poor diff.) & 7.61 & $1.79-32.44$ & 0.006 \\
\hline CEA $(\leq 6$ vs. $>6)$ & 0.40 & $0.14-1.17$ & 0.090 \\
\hline mGPS (0 vs. 1,2) & 5.01 & $1.60-15.69$ & 0.005 \\
\hline
\end{tabular}

levels were measured by turbidimetric immunoassays using an N-Assay TIA CRP-S kit (Nittobo Medical, Tokyo, Japan). Since the limit of detection of this CRP assay was lower than that in other studies $(0.2 \mathrm{vs} .>0.5 \mathrm{mg} / \mathrm{dl})(7,8)$, the cut-off value for abnormal elevation of serum CRP was set at $0.5 \mathrm{mg} / \mathrm{dl}$.

This study aimed to determine whether the mGPS provides more accurate prognostic information than that offered by 
existing staging systems or tumor markers, such as CEA, in stage II/III colorectal cancer patients. In fact, we showed that mGPS was significantly associated with serosal invasion, pre-operative CEA level and TNM classification, which are established conventional prognostic factors. Furthermore, mGPS-positive was found to have independent prognostic value, whereas the prognostic values of CEA or TNM classification were influenced by other clinical factors.

Recent studies on various types of malignancies have emphasized the importance of examining multiple lymph nodes in determining prognosis. In colon and rectal cancer, staging accuracy and survival are improved by increasing the number of nodes examined and analyzed (28-30). In addition, the failure to examine a sufficient number of lymph nodes may result in the inability to identify patients in whom lymph nodes are affected by cancer, thus resulting in understaging (31). However, the number of lymph nodes reported with colectomy varies widely and may be due to variations in surgical technique, the thoroughness of the pathologist in finding nodes in the specimen, or the actual number of regional lymph nodes. Our study showed that high mGPS was the strongest prognostic factor for stage II and III colorectal cancer, rather than lymph node metastasis, which is routinely addressed for postoperative adjuvant chemotherapy. Our results suggest that an objective evaluation method using the mGPS will identify candidates for postoperative chemotherapy to improve poor prognosis revealed by pathological staging.

Surgical resection is highly effective for stage II colorectal cancer, but a significant proportion of these patients (25-30\%) develop recurrence and succumb to the disease. Therefore, identifying sensitive prognostic markers in this subgroup of patients would prompt the use of postoperative adjuvant in these patients with poor prognosis and thus improve survival. In the present study, we demonstrated that pre-operative mGPS was an independent prognostic factor for patients with stage II colorectal cancer. This ability to identify patients with stage II colorectal cancer with a poor prognosis and who would benefit from adjuvant therapy to prevent recurrence could improve cancer survival. In fact, mGPS-positive patients showed a significantly worse prognosis compared to mGPS-negative patients when adjuvant chemotherapy was not used, while cancer-specific survival was improved in the mGPS-positive patients with the worst prognosis when adjuvant chemotherapy was used.

The mechanism of CRP up-regulation is controlled by cytokines, including IL-8, IL-6 and tumor necrosis factor- $\alpha$ (32). We previously investigated host-tumor interactions in patients with colorectal cancer focusing on the defective immunoinflammatory adaptation system against intrinsic IL-6. We reported that an increased serum level of CRP in patients with colorectal cancer reflected, not only enhanced tumor expression of IL-6, but also activation of the interaction between IL- 6 and the IL- 6 receptor in tumor cells, which maintained an active proliferative state $(12,33,34)$. In addition, the systemic antagonistic response against circulating IL-6 derived from the tumor component was found to be suppressed in malnourished cancer patients, which in turn up-regulated IL-6-related systemic induction of CRP (35). IL-6 exerts its action on target cells by acting through a receptor complex consisting of the IL-6 soluble receptor (sR) and a signal- transducing subunit (gp130). IL-6sR/IL-6 complexes play a positive role in local inflammatory reactions by activating cells through membrane-bound gp130. In our previous study, surgery-induced stress increased the formation of IL-6sR/ IL-6 complexes in the operative field, which systemically enhanced IL-6 activity in malnourished colorectal cancer patients (36). Since CRP is known to promote a loss of the membrane-bound IL-6 receptor by proteolytic shedding resulting in a 3-fold increase in IL-6sR production (37), CRP may then up-regulate IL-6-mediated inflammatory events by enabling the formation of the IL-6sR/IL-6 complex in patients with colorectal cancer.

Taken together, the results of the previous and present studies suggest that CRP is more than just an indicator of tumor burden in certain patients with colorectal cancer, in whom tumor burden and a deteriorated host-tumor interaction may synergistically elevate systemic induction of CRP. Elevated CRP levels may then enable the systemic formation of the IL-6sR/IL-6 complex and up-regulate IL-6-mediated tumor growth factors. The subsequent autocrine/paracrine stimulation of residual tumor cells protects cells from apoptosis and regrowth.

In conclusion, pre-operative mGPS levels may provide valuable prognostic information in patients with stage II and III colorectal cancer, and is independent of the CEA test or pathological $\mathrm{N}$ classification system. Accordingly, the mGPS may provide valuable information concerning specific subgroups of patients who might benefit from adjuvant chemotherapy for stage II colorectal cancer. However, further studies are required to confirm our results.

\section{References}

1. Haller DG: An overview of adjuvant therapy for colorectal cancer. Eur J Cancer 31A: 1255-1263, 1995.

2. Wein A, Hahn EG, Merkel S and Hohenberger W: Adjuvant chemotherapy for stage II colon cancer. Eur J Surg Oncol 26: 730-732, 2000

3. Figueredo A, Charette ML, Maroun J, Brouwers MC and Zuraw L: Adjuvant therapy for stage II colon cancer: a systematic review from the Cancer Care Ontario Program in evidence-based care's gastrointestinal cancer disease site group. J Clin Oncol 22: 3395-3407, 2004.

4. Gill S, Loprinzi CL, Sargent DJ, et al: Pooled analysis of fluorouracil-based adjuvant therapy for stage II and III colon cancer: Who benefits and by how much? J Clin Oncol 22: 1797-1806, 2004.

5. Canna K, McMillan DC, McKee RF, McNicol AM, Horgan PG and McArdle CS: Evaluation of a cumulative prognostic score based on the systemic inflammatory response in patients undergoing potentially curative surgery for colorectal cancer. $\mathrm{Br} \mathrm{J}$ Cancer 90: 1707-1709, 2004.

6. Nozoe T, Matsumata T, Kitamura $M$ and Sugimachi K: Significance of preoperative elevation in serum C-reactive protein as an indicator for prognosis in colorectal cancer. Am J Surg 176: 335-338, 1998.

7. McMillan DC, Canna K and McArdle CS: Systemic inflammatory response predicts survival following curative resection for colorectal cancer. Br J Surg 90: 215-219, 2003.

8. Crozier JE, McKee RF, McArdle CS, et al: The presence of a systemic inflammatory response predicts poorer survival in patients receiving adjuvant 5-FU chemotherapy following potentially curative resection for colorectal cancer. Br J Cancer 94: 1833-1836, 2006

9. McMillan DC, Wotherspoon HA, Fearon KC, Sturgeon C, Cooke TG and McArdle CS: A prospective study of tumor recurrence and the acute-phase response after apparently curative colorectal cancer surgery. Am J Surg 170: 319-322, 1995. 
10. Nozoe T, Matsumata T and Sugimachi K: Preoperative elevation of serum C-reactive protein is related to impaired immunity in patients with colorectal cancer. Am J Clin Oncol 23: 263-266, 2000.

11. Gunter MJ, Stolzenberg-Solomon R, Cross AJ, et al: A prospective study of serum $\mathrm{C}$-reactive protein and colorectal cancer risk in men. Cancer Res 66: 2483-2487, 2006.

12. Miki C, Konishi N, Ojima E, Hatada T, Inoue Y and Kusunoki M: $\mathrm{C}$-reactive protein as a prognostic variable that reflects uncontrolled up-regulation of the IL-1-IL-6 network system in colorectal carcinoma. Dig Dis Sci 49: 970-976, 2004.

13. Koike Y, Miki C, Okugawa Y, et al: Preoperative C-reactive protein as a prognostic and therapeutic marker for colorectal cancer. J Surg Oncol 98: 540-544, 2008.

14. Kawano M, Hirano T, Matsuda T, et al: Autocrine generation and requirement of BSF-2/IL-6 for human multiple myelomas. Nature 332: 83-85, 1988.

15. Longo WE, Virgo KS, Johnson FE, et al: Outcome after proctectomy for rectal cancer in Department of Veterans Affairs Hospitals: a report from the National Surgical Quality Improvement Program. Ann Surg 228: 64-70, 1998.

16. Heys SD, Walker LG, Deehan DJ and Eremin OE: Serum albumin: a prognostic indicator in patients with colorectal cancer. J R Coll Surg Edinb 43: 163-168, 1998.

17. Longo WE, Virgo KS, Johnson FE, et al: Risk factors for morbidity and mortality after colectomy for colon cancer. Dis Colon Rectum 43: 83-91, 2000.

18. Forrest LM, McMillan DC, McArdle CS, Angerson WJ and Dunlop DJ: Evaluation of cumulative prognostic scores based on the systemic inflammatory response in patients with inoperable non-small-cell lung cancer. Br J Cancer 89: 1028-1030, 2003.

19. Forrest LM, McMillan DC, McArdle CS, Angerson WJ, Dagg K and Scott HR: A prospective longitudinal study of performance status, an inflammation-based score (GPS) and survival in patients with inoperable non-small-cell lung cancer. Br J Cancer 92: 1834-1836, 2005.

20. Al Murri AM, Bartlett JM, Canney PA, Doughty JC, Wilson C and McMillan DC: Evaluation of an inflammation-based prognostic score (GPS) in patients with metastatic breast cancer. Br J Cancer 94: 227-230, 2006.

21. Crumley AB, McMillan DC, McKernan M, McDonald AC and Stuart RC: Evaluation of an inflammation-based prognostic score in patients with inoperable gastro-oesophageal cancer. $\mathrm{Br} \mathrm{J}$ Cancer 94: 637-641, 2006.

22. Glen P, Jamieson NB, McMillan DC, Carter R, Imrie CW and McKay CJ: Evaluation of an inflammation-based prognostic score in patients with inoperable pancreatic cancer. Pancreatology 6 : $450-453,2006$
23. Ishizuka $M$, Nagata $H$, Takagi $K$, Horie $T$ and Kubota $K$ Inflammation-based prognostic score is a novel predictor of postoperative outcome in patients with colorectal cancer. Ann Surg 246: 1047-1051, 2007.

24. Roxburgh CS, Crozier JE, Maxwell F, et al: Comparison of tumour-based (Petersen Index) and inflammation-based (Glasgow Prognostic Score) scoring systems in patients undergoing curative resection for colon cancer. Br J Cancer 100: 701-706, 2009.

25. Wiggers T, Arends JW, Schutte B, Volovics L and Bosman FT: A multivariate analysis of pathologic prognostic indicators in large bowel cancer. Cancer 61: 386-395, 1988.

26. Fletcher RH: Carcinoembryonic antigen. Ann Int Med 104: 66-73, 1986.

27. Moertel CG, Fleming TR, Macdonald JS, Haller DG, Laurie JA and Tangen C: An evaluation of the carcinoembryonic antigen (CEA) test for monitoring patients with resected colon cancer. JAMA 270: 943-947, 1993

28. Tepper JE, O'Connell MJ, Niedzwiecki D, et al: Impact of number of nodes retrieved on outcome in patients with rectal cancer. J Clin Oncol 19: 157-163, 2001

29. Joseph NE, Sigurdson ER, Hanlon AL, et al: Accuracy of determining nodal negativity in colorectal cancer on the basis of the number of nodes retrieved on resection. Ann Surg Oncol 10: 213-218, 2003

30. Joseph NE, Sigurdson ER, Hanlon AL, et al: Colon cancer survival is associated with increasing number of lymph nodes analyzed: A secondary survey of intergroup trial INT-0089. J Clin Oncol 21: 2912-2919, 2003

31. Swanson RS, Compton CC, Stewart AK and Bland KI: The prognosis of T3N0 colon cancer is dependent on the number of lymph nodes examined. Ann Surg Oncol 10: 65-71, 2003.

32. Nakazaki H: Preoperative and postoperative cytokines in patients with cancer. Cancer 70: 709-713, 1992.

33. Wakuda R, Miki C and Kusunoki M: Autoreactivity against interleukin 6 as a risk factor in elderly patients with colorectal carcinoma. Arch Surg 136: 1274-1279, 2001.

34. Kinoshita T, Ito $\mathrm{H}$ and Miki C: Serum interleukin-6 level reflects the tumor proliferative activity in patients with colorectal carcinoma. Cancer 85: 2526-2531, 1999.

35. Miki C, Inoue Y, Toiyama Y, et al: Deficiency in systemic interleukin-1 receptor antagonist production as an operative risk factor in malnourished elderly patients with colorectal carcinoma. Crit Care Med 33: 177-180, 2005.

36. Hatada $\mathrm{T}$ and Miki $\mathrm{C}$ : Nutritional status and postoperative cytokine reponse in colorectal cancer patients. Cytokine 12 1331-1336, 2000.

37. Jones SA, Novick D, Horiuchi S, Yamamoto N, Szalai AJ and Fuller GM: C-reactive protein: a physiological activator of interleukin 6 receptor shedding. J Exp Med 189: 599-604, 1999. 